ECE2015

Dublin, Ireland

16 - 20 May 2015

EP-669

\section{MiR-7 has a role in behaviour of somatotroph adenomas}

Ruth Sánchez-Ortiga1 ${ }^{1}$ Laura Sánchez-Tejada², Cristina Lamas ${ }^{3}$, Rosa Cámara ${ }^{4}$, Javier Abarca ${ }^{5}$, Irene Monjas ${ }^{6}$, Pedro Riesgo $^{7}$, Carmen Fajardo ${ }^{8}$, Antonio M. Picó Alfonso ${ }^{1}$

1 Endocrinology Department, Hospital General Universitario Alicante, Spain. 2 Research Unit of Endocrinology Department, Hospital General Universitario Alicante, Spain. 3 Endocrinology Department H.G.U. Albacete, Spain. 4 Endocrinology Department H.U. La Fé de Valencia, Spain. 5 Neurosurgery Department, Hospital General Universitario Alicante, Spain. 6 Otorhinolaryngology Department, Hospital General Universitario Alicante, Spain. 7 Neurosurgery Department, Hospital La Ribera, Spain. 8 Endocrinology Department, Hospital La Ribera, Spain.

Insulin-like growth factor receptor 1 (IGF1R) and epidermal growth factor receptor (EGFR) are receptors tyrosine-kinase (RTK) whose altered signaling is critical in the development of many types of tumors. These RTK are some of the main targets of the microRNA (miRNA) miR-7. miR-7 is an important tumor suppressor miRNA. It has the ability to inhibit the motility, invasiveness and anchorageindependent growth, suggesting a strong therapeutic potential in many types of cancer. Pituitary adenomas (PA) are a heterogeneous group of tumors with diverse clinical behaviour in whom the study of miRNA network has scarcely studied.

The aim of this study was to investigate the role of miR-7 in the behaviour of different PA subtypes.

\title{
Methods
}

In this cross-sectional descriptive study, we evaluated miR-7 by qRT-PCR on 60 human PA: 29 gonadotrophs (GT), 15 somatotrophs (ST), 8 functioning corticotroph (CT) and 8 silent corticotroph adenomas (SCA). 9 healthy pituitary from autopsies were used as calibrator reference. We graded aggressiveness according to invasiveness and Ki-67 gene expression: high:Hardy's grade IV and Ki$67>2.59$ fold change (FC); medium: Hardy's grade IV or Ki-67 >2.59 FC, and low: Hardy's grade <IV and Ki-67 <2.59 FC.

\section{Results}

MiR-7 showed different expression levels depending on PA subtype, with its lower expression in CT (figure 1). MiR-7 expression patterns showed also significative differences depending on PA subtype $(p(K-W)=0.002)$, although they were similar in non-functioning tumors (GT and SCA) (figure 2). Even though ST were the adenomas with highest expression of mir-7 (figure 3 ), in $33 \%$ of ST the expression was repressed. This miR-7 repression entailed a risk of aggressiveness of $7(2-25)$ times (px2=0.038). We did not find associations with tumour extension or aggressiveness in the other PA subtypes.

Figure 2. miR-7 expression patterns in PA subtypes

Figure 1. miR-7 expression levels in PA subtypes

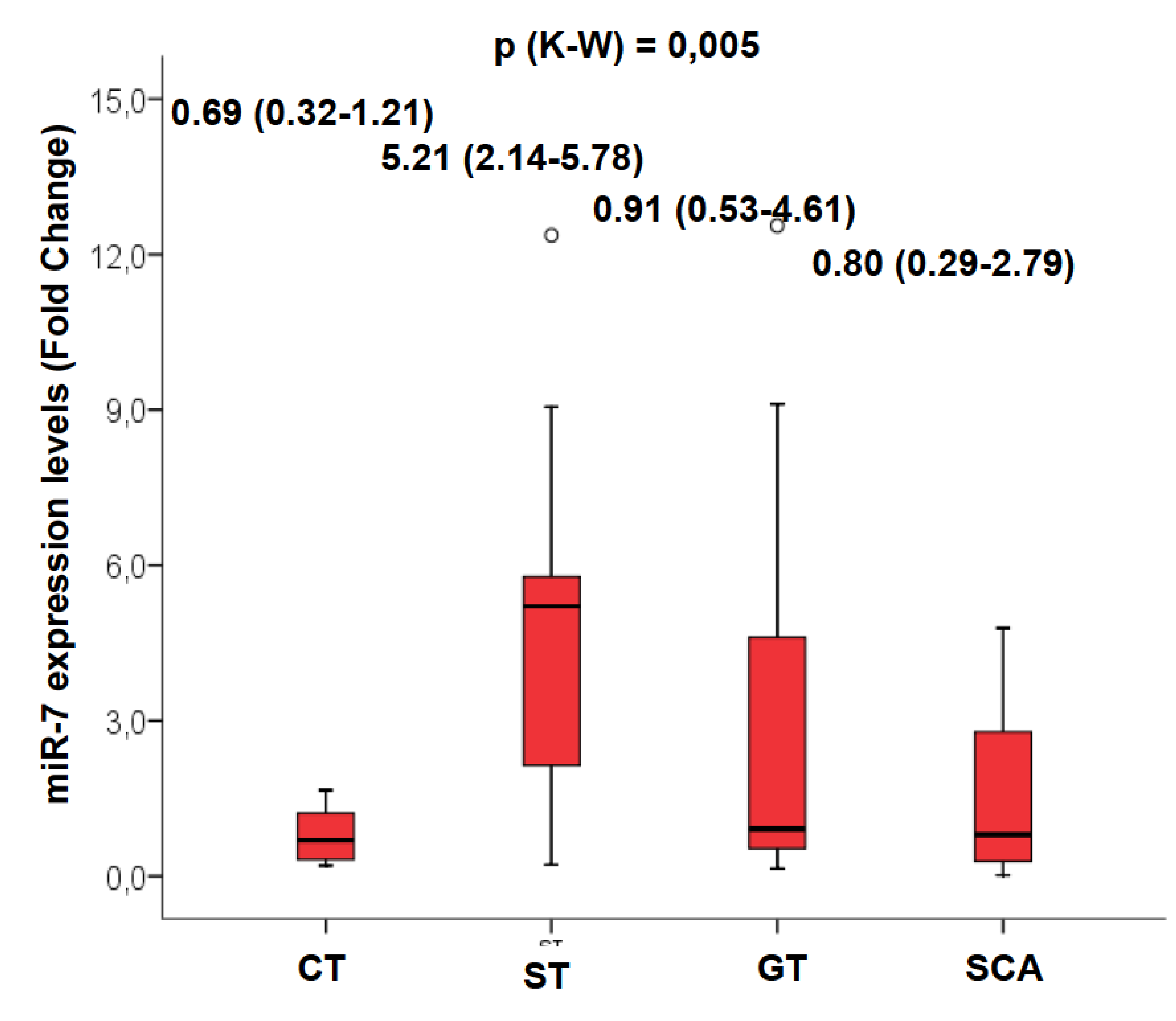

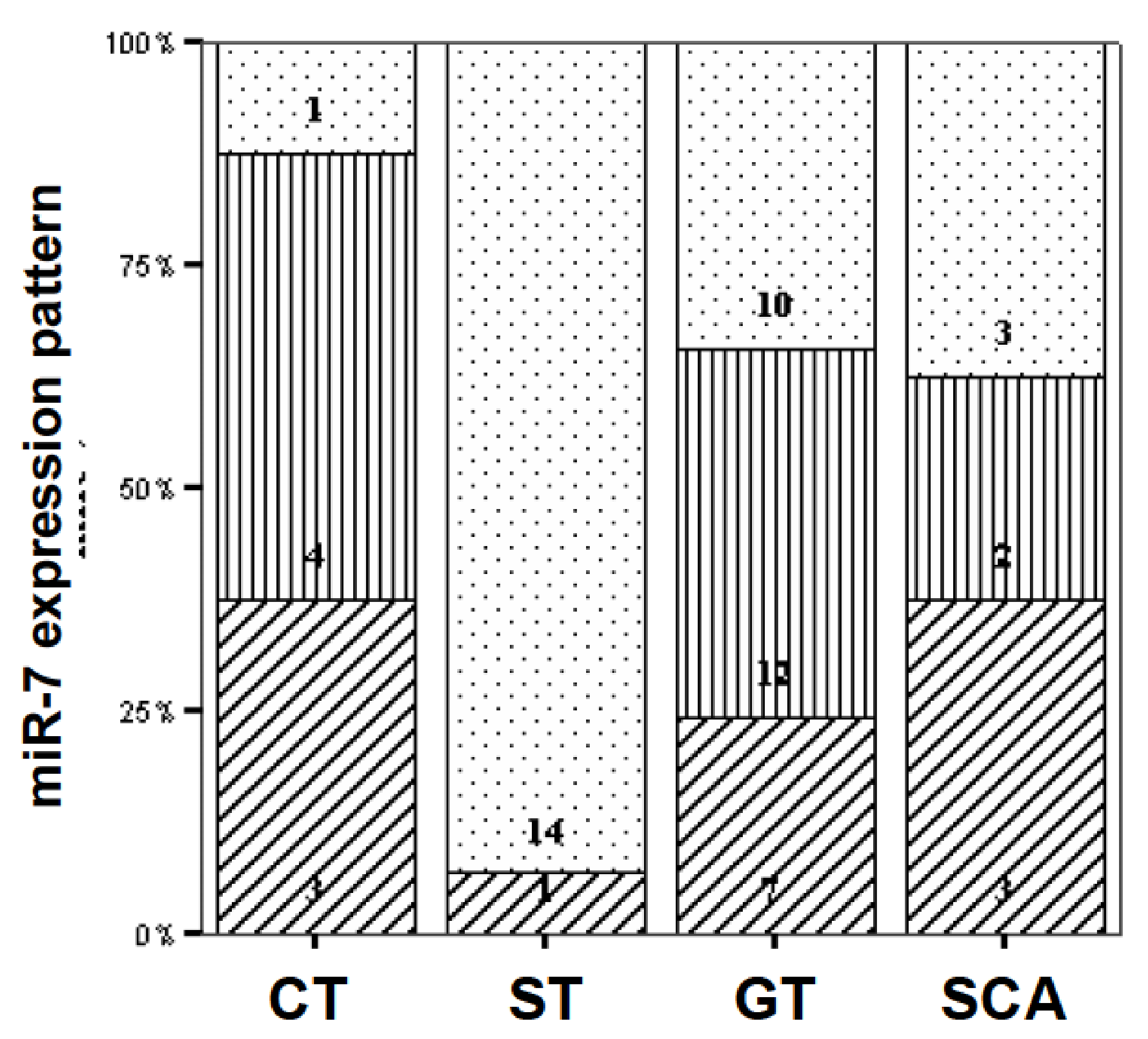

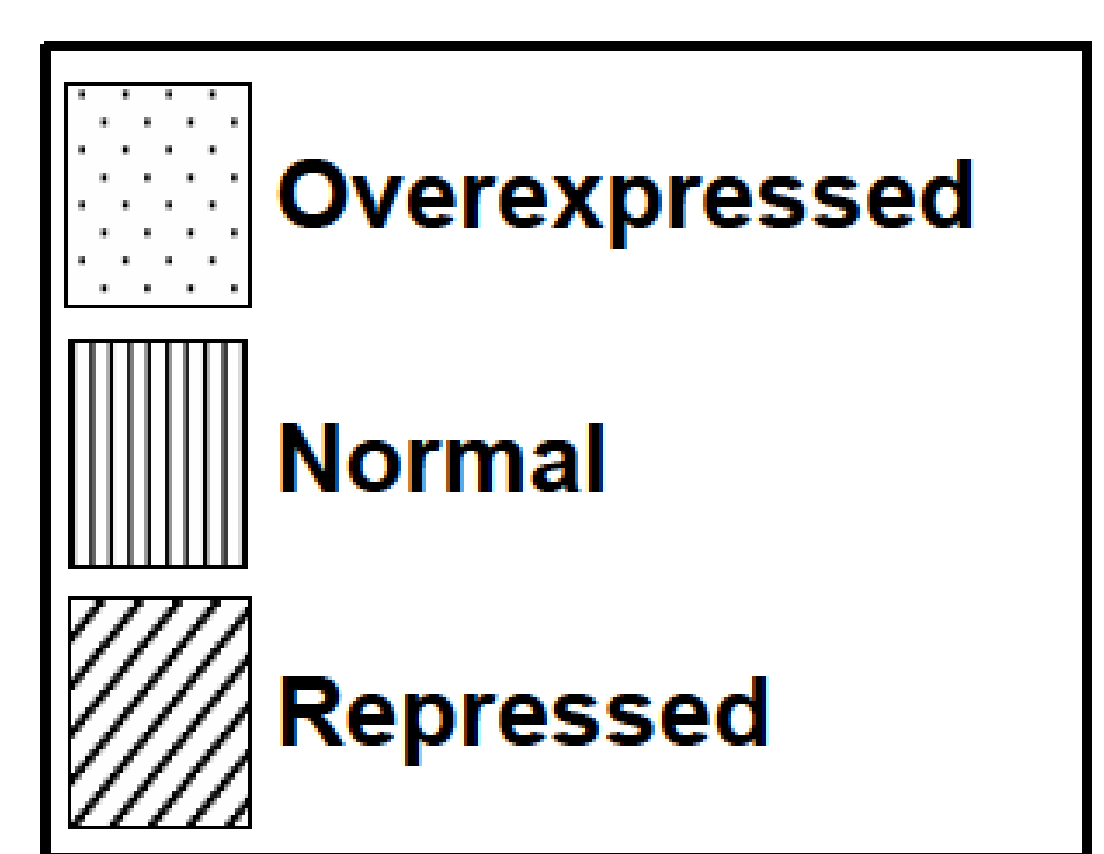

Figure 3. miR-7 expression (fold change) in ST

$$
\begin{array}{cc}
\mathrm{p}(\mathrm{MWU})= & 0,001 \\
5.21(2.14-5.78) & 0.88(0.37-2.22)
\end{array}
$$

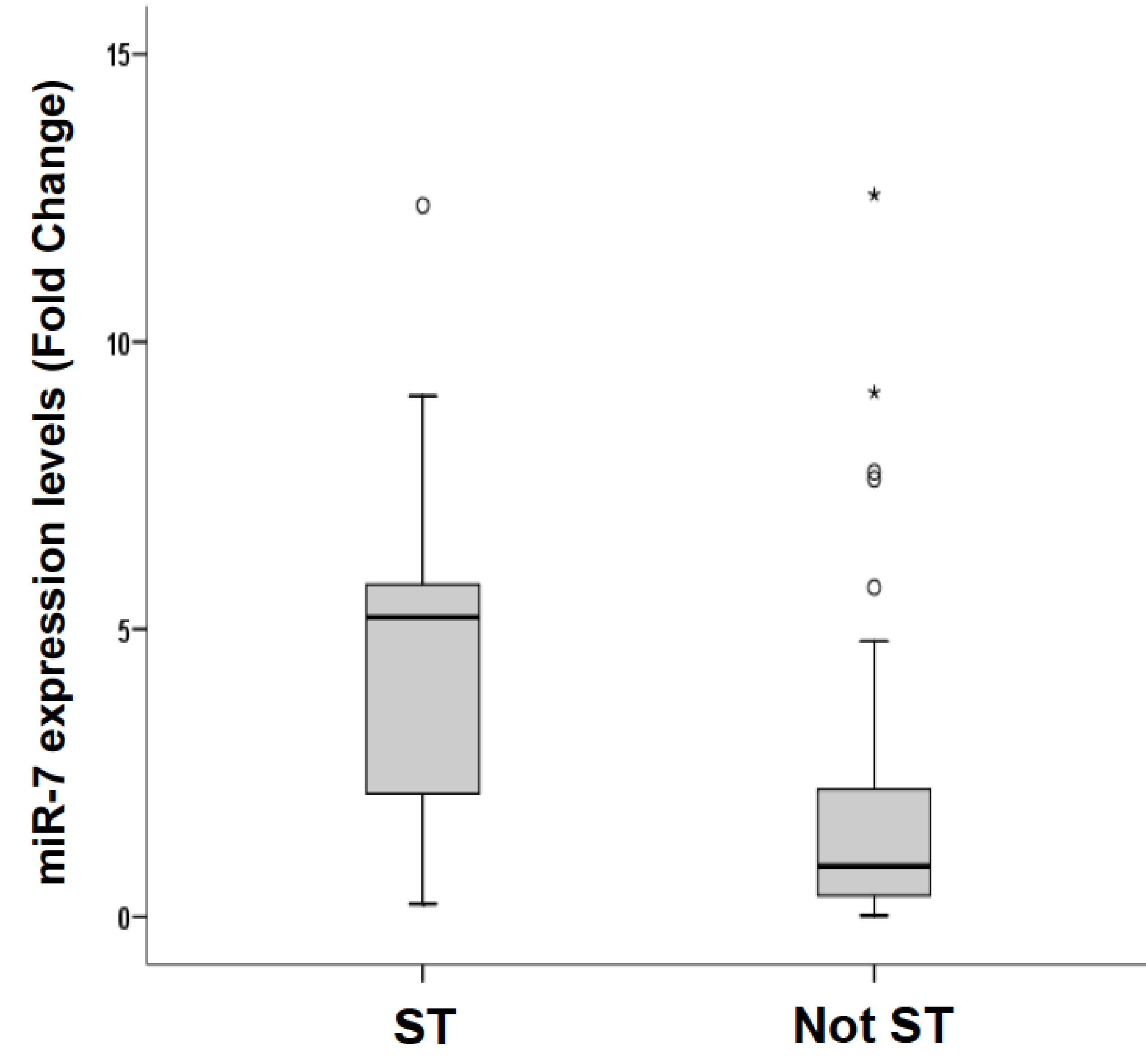

\section{Conclusions}

According with our results, miR-7 plays an important role in the behaviour of ST, acting as tumor suppressor miRNA, probably contributing to control its growth. That open the door to a new potential therapeutic approach. 